\title{
Measurement of Enhanced Radiation Force on a Parallel Metallic-Plate System in the Microwave Regime
}

\author{
Z. Marcet, ${ }^{1,2,3}$ Z. H. Hang, ${ }^{1,4}$ S. B. Wang, ${ }^{1,2}$ J. Ng, ${ }^{5}$ C. T. Chan, ${ }^{1,6}$ and H. B. Chan ${ }^{1,2, *}$ \\ ${ }^{1}$ Department of Physics, The Hong Kong University of Science and Technology, Clear Water Bay, Kowloon, Hong Kong, China \\ ${ }^{2}$ William Mong Institute of Nano Science and Technology, The Hong Kong University of Science and Technology, \\ Clear Water Bay, Kowloon, Hong Kong, China \\ ${ }^{3}$ Department of Physics, University of Florida, Gainesville, Florida 32611, USA \\ ${ }^{4}$ School of Physical Science and Technology, Soochow University, 1 Shizi Street, Suzhou 215006, China \\ ${ }^{5}$ Department of Physics and Institute of Computational and Theoretical Studies, Hong Kong Baptist University, \\ Kowloon Tong, Hong Kong, China \\ ${ }^{6}$ Institute for Advanced Study, The Hong Kong University of Science and Technology, \\ Clear Water Bay, Kowloon, Hong Kong, China
}

(Received 8 November 2013; published 28 January 2014)

\begin{abstract}
We measure the force exerted by microwave radiation on a centimeter-sized parallel-plate metallic resonant unit. By varying the ambient environment, we distinguish carefully between the direct radiation force and the indirect photothermal component. At the microwave resonance, the former is measured quantitatively to be 100 times larger than the conventional radiation force. Furthermore, the enhanced radiation force tends to increase the separation of the plates. Both the direction and the magnitude of the measured force agree well with numerical calculations.
\end{abstract}

Artificially structured materials such as metamaterials have demonstrated a remarkable ability to manipulate electromagnetic (EM) radiation in manners not possible with conventional media. Due to the resonant coupling of the resonating building blocks of these materials with EM fields, novel effects such as negative refraction [1,2], superimaging [3-5], and cloaking $[2,6]$ have been achieved from the optical to microwave parts of the spectrum. One promising area of progress involves modifying the properties of the resonating units using EM radiation to construct tunable or reconfigurable systems. By incorporating elastically deformable elements [7], it is possible to design resonators whose structure can be controlled by the mechanical impact or the heating effects of the incident EM radiation. The tunable changes in the resonant wavelength can be exploited in a wide range of applications, such as reconfigurable [8-11] and nonlinear [12,13] metamaterials.

At resonance, the EM fields in metamaterials and plasmonic structres are strongly concentrated at specific locations. As a result, the electromagnetically induced forces on the resonating units can be orders of magnitude larger than the conventional photon force on an ordinary reflecting surface [14-21]. Measurement or exploitation of the enhanced electromagnetic forces in metamaterials, however, is nontrivial because the strong currents induced in the metallic resonating elements also lead to heating. The mechanical deformations arising from such thermal effects are commonly associated with photothermal forces. In conventional materials, separating the effects of the EM force and the photothermal force often relies on the different time response of the mechanical structures [22]. Unlike the
EM force that is exerted almost immediately on the structure as the radiation is turned on, there exists a time delay for the photothermal force. When the intensity of the incident EM radiation is time modulated at a frequency that is much higher than the rate of thermal relaxation, the periodic mechanical response to the photothermal force becomes negligible compared to the EM force, allowing these two effects to be separated from each other. For metamaterials, measurement of the enhanced EM force exerted on the resonating units, to our knowledge, has not yet been reported.

In this Letter, we measure the force exerted by microwave radiation on an EM resonating system consisting of centimeter-sized metallic parallel plates, in which the top plate is suspended by springs. The mechanical oscillations of the movable plate in response to the amplitudemodulated microwave radiation are detected capacitively. We carefully distinguish the direct EM force from the photothermal force. At the microwave resonance of $\sim 14.5 \mathrm{GHz}$, the EM force induced on the top plate is observed to be directed away from the metalized substrate. At an incident microwave power of $200 \mathrm{~mW}$, the magnitude of the force is measured to be $60 \mathrm{nN}, \sim 100$ times larger than the conventional radiation force. Both the direction and the magnitude of the measured force agree well with numerical calculations.

Figure 1(a) shows the parallel-plate resonant unit used in experimental measurements. It consists of a gold-coated nickel plate $(1 \mathrm{~cm}$ by $1 \mathrm{~cm}$ in area and $25 \mu$ m thick) suspended above a gold-coated silicon substrate by two springs $(5 \mathrm{~mm}$ by $1 \mathrm{~mm}$ by $25 \mu \mathrm{m}$ ) centered on opposite sides of the plate. The upper metal plate and the metal-coated substrate forms a 

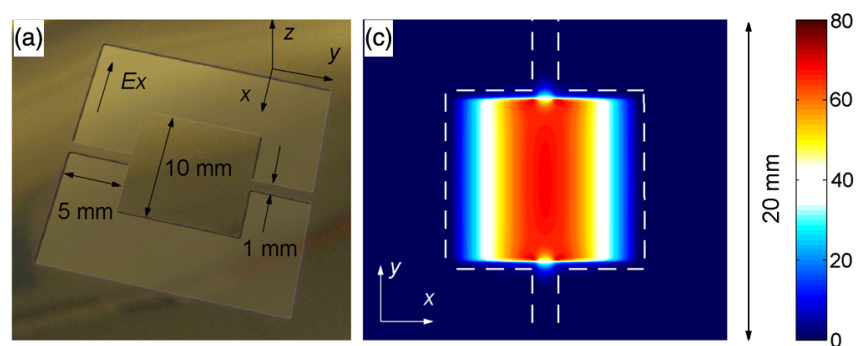

(b)

(d)
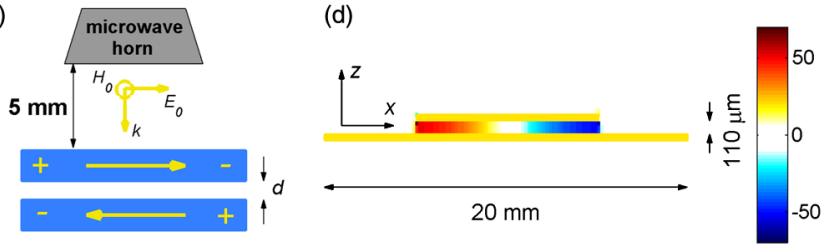

FIG. 1 (color online). (a) Top view optical image of the parallelplate resonant cavity. The square top plate in the middle is suspended by two springs. (b) Schematic cross section of the structure showing the charge accumulation and antiparallel induced current at microwave resonance. The magnetic (c) and electric (d) field distributions in the gap at microwave resonance are shown with the magnitude represented by the color scales. The magnetic field points along $y$ in (c) and the electric field points along $z$ in (d). The microwave beam is incident along $-z$ with unit amplitude, with electric field along $x$.

cavity to confine the EM wave. The initial gap between the movable plate and the substrate is $110 \mu \mathrm{m}$, set by an SU8 layer that is lithographically patterned to form spacers at the periphery of Fig. 1(a) on which the frame of the suspended plate is placed. As shown in Fig. 1(b), a microwave horn is positioned on top of the resonant cavity. It emits microwave with the electric field, magnetic field, and the wave vector pointing along the $x, y$ and $z$ directions, respectively. In this configuration, a radiation pressure is exerted in the direction normal to the movable plate. The resonant cavity and microwave horn are both placed inside a bell jar that is evacuated to pressures lower than $9 \times 10^{-3}$ torr.

The EM energy stored between the metal plates attains a maximum at microwave resonance. In the cross sectional schematic shown in Fig. 1(b), we consider the antisymmetric mode in which the time-varying magnetic flux (normal to the page) leads to a periodic oscillation of current that flows along opposite directions in the top and bottom plates. The current oscillations are accompanied by the periodic buildup of positive and negative charges at the left and right ends of the plates [Fig. 1(b)]. As a result, both magnetic and electric forces are generated on the plates. The antiparallel induced currents lead to a repulsive magnetic force between the plates. Due to the current flow, opposite charges accumulate periodically at the ends of the two plates, resulting in an electric Coulomb attraction. The net time-averaged total force is the sum of the repulsive magnetic and attractive electric forces. For a parallel plate system, it can be proven that repulsive magnetic and attractive electric forces tend to cancel each other as the size of the plate increases to infinity [17]. However, for a finite-sized parallel-plate structure, the electric field leaks out at the edges of the cavity, reducing the magnitude of the electrostatic attraction. In contrast, the magnetic field is concentrated at the center of the cavity and the magnetic repulsion is therefore much less susceptible to edge effects. As a result, the incident microwave generates a net repulsive force between the movable plate and the substrate. Figures 1(c) and 1(d) show, respectively, the calculated induced magnetic and electric field distribution at microwave resonance. The field amplitude in the cavity is enhanced by up to 80 times relative to the incident microwave. At resonance, the magnitude of the induced EM pressure on the top gold plate exceeds the conventional radiation pressure by a factor of $\sim 100$. Similar enhancement of radiation pressure has been theoretically predicted for a simpler two-dimensional case [17].

It is instructive to distinguish the aforementioned mechanism for microwave radiation pressure enhancement to that in Fabry-Perot cavities in optomechanics. In the latter, the radiation pressure on the mirrors is enhanced as the photon bounces back and forth many times between the mirrors. Typically, the separation between the mirrors is much larger than the wavelength of light. The condition for resonance is satisfied when the separation is an integer multiple of half wavelengths. In our system, the underlying mechanism is fundamentally different as the effect arises due to the excitation of coupled resonances [16-18] on the parallelplate system. The gap between the two plates is about a factor of 200 smaller than the resonant wavelength, and the dominant factor in determining the resonant wavelength is the size of the plate rather than the gap between the plates.

Motion of the top plate can be described by the equation

$$
\ddot{z}+\gamma \dot{z}+\omega_{0}^{2} z=F / m
$$

where $z$ is the displacement out of the substrate, $m$ is the mass, $\gamma$ is the damping constant, $\omega_{0}=\sqrt{k / m}$ $\left(1445 \mathrm{rad} \mathrm{s}^{-1}\right)$ is the natural frequency for the mechanical oscillations, and $k$ is the spring constant. For the different gas pressures used, the quality factor of the mechanical vibrations ranges from 29 to 115 . The external applied force $F$ consists of three components,

$$
F=F_{\mu \text { wave }}+F_{\text {thermal }}+F_{\text {electrostatic }},
$$

$F_{\mu \text { wave }}$ is the electromagnetic force associated with the current and charge buildup induced by the microwave as described earlier, time-averaged over a microwave period. $F_{\text {thermal }}$ is the photothermal force, which originates from the Ohmic heating due to current induced by the microwave, as we will discuss in detail later. Both $F_{\mu \text { wave }}$ and $F_{\text {thermal }}$ are proportional to the microwave intensity. The last term, $F_{\text {electrostatic }}$, represents the electrostatic force that is used for calibration. When a potential difference $V$ is applied between the top plate and the substrate via the conductive 
springs, an attractive force with known magnitude is generated. In Eq. (2), each term contains a dc component and a periodic modulation with frequency ranging from $\sim 0.1 \omega_{0}$ to $\sim 100 \omega_{0}$. For a chosen microwave frequency, the dc components of all the forces are kept constant. In the following analysis, we focus on the periodic components.

Vibrations of the top plate are detected capacitively through induced modulations on a carrier voltage signal. When the top plate is displaced in response to external forces, the capacitance $C_{x}$ between the top plate and the substrate changes. As shown in Fig. 2, two ac voltages, $V_{c 1}$ and $V_{c 2}$, of the same frequency $\left(f_{c}=100 \mathrm{kHz} \gg \omega_{0} / 2 \pi\right)$ and amplitude $(100 \mathrm{mV})$ but opposite phase are applied to the bottom plate and one plate of a fixed capacitor $C_{2}$ that is chosen to be close to $C_{x}$. The top plate and the other plate of $C_{2}$ are connected. As the top plate vibrates, the initial gap $d$ is increased by an amount $z$, leading to changes in $C_{x}$. As a result, the amplitude of the carrier signal on the top plate is modulated by the plate motion. The voltage on the top plate is measured with a lock-in amplifier referenced to $f_{c}$, yielding an output $V_{\text {signal }}$ that is proportional to the displacement $z$, with a proportionality constant $\alpha$. We define $z$ to be positive when the top plate moves away from the substrate.

To find the proportionality constant $\alpha$ that relates voltage to displacement, we add a voltage $V_{d c}+V_{a c} \cos (\omega t)$ between the two plates, where $V_{d c}(0.6 \mathrm{~V})$ is much larger than $V_{a c}(0.1 \mathrm{~V})$ and $\omega \sim \omega_{0}$. The periodic electrostatic force generated is given by $F_{\text {electrostatic }}=F_{e} \cos (\omega t)$, where $F_{e}=-\epsilon V_{d c} V_{a c} A / d^{2}$, with the negative sign indicating that the force is directed towards the substrate. The displacement of the top plate in response to a periodic force with time dependence of $\cos (\omega t)$ can be written as

$$
z=P \cos (\omega t)-Q \sin (\omega t) .
$$

If the external force $F$ only has the $F_{\text {electrostatic }}$ component, Eq. (1) yields

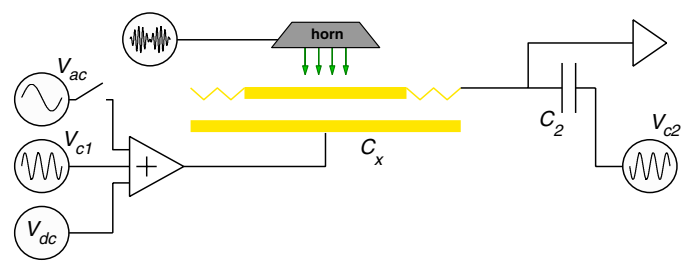

FIG. 2 (color online). Motion of the top plate is detected capacitively. Changes in $C_{x}$ are measured by a capacitance bridge with carrier ac voltages $V_{c 1}$ and $V_{c 2}$ at $100 \mathrm{kHz}$. During electrostatic calibration, an ac voltage $V_{a c} \cos (\omega t)$ is added to the bottom plate and the microwave modulation frequency is increased to $\sim 100 \omega_{0}$, so that mechanical vibrations are solely excited by the electrostatic force. In the detection of the EM force due to the microwave, the electrostatic force is turned off and the microwave modulation frequency is chosen to be $\sim \omega_{0}$ to excite the mechanical resonance.

$$
P+i Q=F_{e} / m /\left(\omega_{0}^{2}-\omega^{2}+i \gamma \omega\right),
$$

which shows that measuring $P(\omega)$ and $Q(\omega)$ can determine mechanical properties of the oscillator system as characterized by $\omega_{0}$ and $\gamma$. Figure 3(a) plots $P$ and $Q$ as a function of $\omega$, yielding fitted values of $\omega_{0}$ and $\gamma$ of $1440 \mathrm{rad} \mathrm{s}^{-1}$ and $85.1 \mathrm{rad} \mathrm{s}^{-1}$, respectively. $\alpha$ is then determined by the ratio of the amplitude of the oscillations in $V_{\text {signal }}$ to the vibration amplitude $R=\sqrt{\left(P^{2}+Q^{2}\right)}$ as calculated with Eq. (4). In the $P-Q$ phase space shown in Fig. 3(b), the red arrow represents the mechanical resonance, where the oscillation amplitude attains maximum and the phase lags behind the periodic electrostatic excitation by $\pi / 2$. As we will describe later, the microwave radiation remains on during this calibration procedure, with a modulation frequency much higher than $\omega_{0}$. Due to the narrow linewidth for mechanical resonance, vibrations induced by the microwave at $\omega_{0}$ are negligible during calibration.

Next, we turn off the periodic electrostatic force by setting $V_{a c}$ to zero and study the forces generated by the microwave radiation. We choose the microwave frequency to be $14.35 \mathrm{GHz}$ and the frequency for amplitude modulation to be close to $\omega_{0}$. The intensity of the microwave is given by $I_{1}+I_{0} \cos (\omega t)$, where $I_{1}$ and $I_{0}$ are time independent. In Fig. 3(b), the blue triangles represent the vibration quadratures $P$ and $Q$ obtained from capacitive detection. Specifically, $V_{\text {signal }}$ measured by the lock-in amplifiers are scaled by the proportionality constant $\alpha$ to yield the plate displacement. The most prominent feature is that the vibrations are out of phase compared to those generated by the electrostatic force. In other words, the
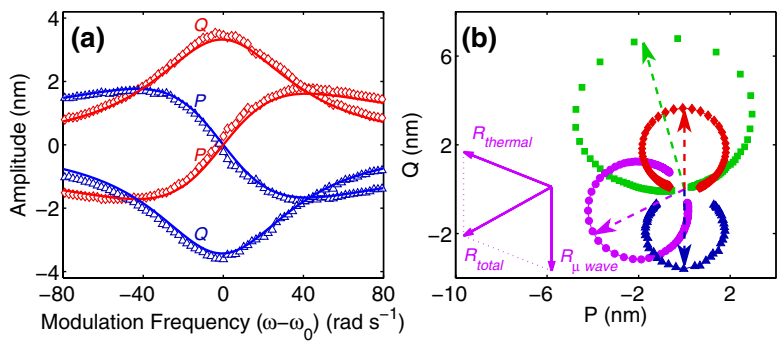

FIG. 3 (color online). (a) The mechanical oscillation quadratures $P$ and $Q$ that are in phase and out of phase with the periodic electrostatic drive (diamonds) and modulated microwave intensity (triangles), respectively, as a function of $\omega$. The lines are fits using Eq. (4). (b) The phase space of $P$ and $Q$. The red diamonds represent mechanical vibrations in response to the periodic electrostatic force [same data as plotted in (a)]. The blue triangles, purple circles, and green squares represent responses to the modulated microwave at microwave resonance, at pressure of $8.6 \times 10^{-3}, 2.5 \times 10^{-4}$, and $2.8 \times 10^{-5}$ torr, respectively. The incident microwave power is $200 \mathrm{~mW}$. The arrows indicate the mechanical resonance where the vibration amplitude attains maximum. Inset: $R_{\mu \text { wave }}$ and $R_{\text {thermal }}$ depict the vibrational response to $F_{\mu \text { wave }}$ and $F_{\text {thermal }}$, respectively, at $2.5 \times 10^{-4}$ torr. $R_{\text {total }}$ is the overall response. 
EM force generated by the microwave is opposite to the electrostatic force, which means that the EM force tends to increase the separation of the plates. Here, the bell jar is kept at $8.6 \times 10^{-3}$ torr. As we will demonstrate below, at this pressure the periodic component of $F_{\text {thermal }}$ is negligible compared to that of $F_{\mu \text { wave }}$.

$F_{\text {thermal }}$ is associated with the Ohmic heating of the current induced by the microwave. The incident microwave leads to a rise of the temperature of the entire parallel plate structure. Due to the difference in thermal expansion of the metal, the SU8 spacer, and the silicon substrate, the springs are deformed. By using a capacitance bridge to measure the capacitance between the top plate and bottom electrode, we take into account the change in the gap $d$ when the device is under microwave illumination. As we mentioned before, we keep the value of $I_{0}$ and $I_{1}$ constant for the measurement of $F_{\text {electrostatic }}$ and $F_{\mu \text { wave }}$. Furthermore, whenever the microwave frequency is changed, we wait for the temperature to stabilize before performing any force measurement. Such a procedure ensures that the proper value of $d$ is used in the calibration of $\alpha$, so that the heating effects are properly accounted for. In Fig. 4, the solid blue squares plot the measured value of $F_{\mu \text { wave }}$ as the microwave frequency is varied from 13.5 to $15 \mathrm{GHz}$. $F_{\mu \text { wave }}$ attains a maximum of $\sim 60 \mathrm{nN}$ at $\sim 14.5 \mathrm{GHz}$, where the induced currents and charges are the strongest due to microwave resonance. The positive values represent the fact that the direction of the forces is out of the substrate. Numerical simulation results of $F_{\mu \text { wave }}$ are shown as the red line, where the changes in $d$ due to the thermal effect at different microwave frequencies are measured and taken into account. The numerical evaluation of $F_{\mu \text { wave }}$ involves two steps. First, the total EM fields are calculated using numerical field solvers $[17,23-25]$ based on the exact geometry of the sample. We then calculate the time-averaged force using the Maxwell stress tensor method [26] as $\langle\mathbf{F}\rangle=\oint_{\partial \Omega}\langle\mathbf{T}\rangle \cdot \hat{n} d A$, where $\overline{T_{i j}}=\epsilon_{0} E_{i} E_{j}+\mu_{0} H_{i} H_{j}-(1 / 2)\left(\epsilon_{0} \mathbf{E} \cdot \mathbf{E}+\mu_{0} \mathbf{H} \cdot \mathbf{H}\right) \delta_{i j}$ are the stress tensor elements and $\langle\cdots\rangle$ stands for time

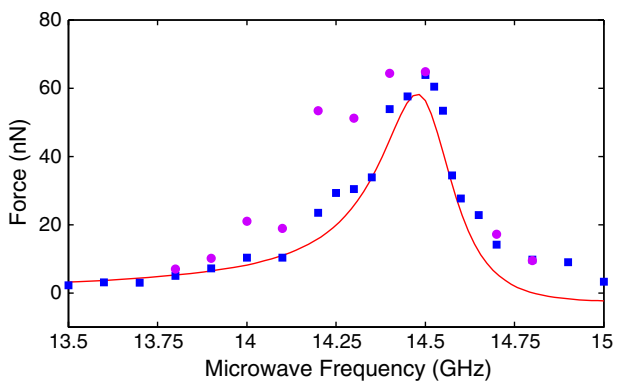

FIG. 4 (color online). $\quad F_{\mu \text { wave }}$ is measured for two values of bell jar pressure at the incident microwave power of $200 \mathrm{~mW}$. The blue squares are measured at $8.6 \times 10^{-3}$ torr where the thermal dissipation is sufficiently high so that $F_{\text {thermal }}$ is negligible. The purple circles are taken at $2.5 \times 10^{-4}$ torr where $F_{\mu \text { wave }}$ and $F_{\text {thermal }}$ are comparable, so that it is necessary to extract $F_{\mu \text { wave }}$ by fitting to Eq. (6). Numerically calculated values are shown by the solid line. averaging. The Maxwell tensor gives the time-averaged total force $\langle F\rangle$ after integrating over a virtual box enclosing the top plate, where the box surface and the unit normal vector are denoted by $\partial \Omega$ and $\hat{n}$, respectively. We note that the Maxwell tensor approach gives the direct radiation force due to the EM fields. The indirect effect due to heating is not included. Measurements agree well with the numerical simulations, accurately matching the position, magnitude, and shape of the microwave resonance. Comparing our measured EM force due to the resonance to the regular radiation force on a perfectly flat conducting surface, we find that the former exceeds the latter by a factor of 100 and the directions are opposite to each other.

In addition to $F_{\mu \text { wave }}$, the amplitude modulation of the microwave $I_{0} \cos (\omega t)$ produces time-periodic Ohmic heating effects that are localized at specific locations of the top plate as determined by the spatial distribution of the induced current. The periodic stress associated with thermal expansion generates the ac component of $F_{\text {thermal }}$. Its sign and magnitude strongly depend on the composition and geometry of the sample. For the blue data points in Fig. 4, the bell jar pressure is maintained at $8.6 \times 10^{-3}$ torr. The thermal conductivity of the gas is sufficiently high so that the contribution of $F_{\text {thermal }}$ is negligible compared to $F_{\mu \text { wave. }}$. If the gas pressure is reduced, $F_{\text {thermal }}$ increases due to the decrease in local thermal conductivity. Even though both $F_{\text {thermal }}$ and $F_{\mu \text { wave }}$ have ac components that are proportional to $I_{0}$, their effects on the top plate can be distinguished from each other from the phase delay of the induced mechanical vibrations. The direct EM force $F_{\mu \text { wave }}$ is generated by the current and charges induced by the microwave. Its dependence on the amplitude modulated microwave is almost instantaneous. In contrast, $F_{\text {thermal }}$ arises due to Ohmic heating that is associated with electronphonon coupling and heat transport. Due to the finite thermal response time of the structure, there exists a time delay between $F_{\text {thermal }}$ and the microwave intensity modulations. The effects of the periodic components of $F_{\text {thermal }}$ on the mechanical vibrations become observable when we lower the gas pressure to reduce the local thermal dissipation. In Fig. 3(b), the purple circles and green squares represent the measured quadratures of oscillations $P$ and $Q$ for bell jar pressures of $2.5 \times 10^{-4}$ and $2.8 \times 10^{-5}$ torr, respectively. As the pressure decreases, the mechanical resonances indicated by the arrows rotate counterclockwise in the two-dimensional phase space. Since $F_{\mu \text { wave }}$ is independent of gas pressure, the phase delays shown in Fig. 3(b) are associated with the effects of $F_{\text {thermal }}$ on the mechanical vibrations that increase with decreasing pressure.

It is possible to distinguish between $F_{\mu \text { wave }}$ and $F_{\text {thermal }}$ if we assume an exponentially delayed impulse response function for the latter, $h(t)=1-\exp \left(-t / t_{0}\right)$. The forces induced by the microwave radiation can be written as [22] 


$$
\begin{aligned}
& F_{\mu \text { wave }}(t)=c_{1} I(t), \\
& F_{\text {thermal }}(t)=\int_{0}^{t} c_{2} \frac{d I\left(t^{\prime}\right)}{d t^{\prime}} h\left(t-t^{\prime}\right) d t^{\prime},
\end{aligned}
$$

where $I(t)$ is the intensity of the microwave. $c_{1}$ and $c_{2}$ are proportionality constants that depend on the microwave frequency, attaining maxima at the microwave resonance. For fixed gas pressure, $t_{0}$ remains constant as the microwave frequency is varied. By substituting Eq. (5) into Eq. (1), the amplitudes of the two quadratures of mechanical vibrations in response to $I(t)=I_{0} \cos (\omega t)$ are given by

$$
P+i Q=I_{0} \frac{c_{1}+c_{2} /\left(1+i \omega t_{0}\right)}{m\left(\omega_{0}^{2}-\omega^{2}+i \gamma \omega\right)}
$$

At high modulation frequency, $\omega t_{0} \rightarrow \infty$, the effect of $F_{\text {thermal }}$ is negligible. For instance, this condition is satisfied during electrostatic calibration where the microwave modulation frequency is set to $20 \mathrm{kHz}$. When $\omega t_{0}$ is small, $1 /\left(1+i \omega t_{0}\right) \approx e^{-i \omega t_{0}}$, representing a relative phase lag between the mechanical response to $F_{\mu \text { wave }}$ and $F_{\text {thermal }}$. The solid purple line in Fig. 3 represents a fit to $P$ and $Q$ at $2.5 \times 10^{-4}$ torr, yielding fitted values of $-40.2 \mathrm{~nm} \mathrm{rad}^{2} \mathrm{~s}^{-2} \mathrm{~W}^{-1} g^{-1}, \quad-46.1 \mathrm{~nm} \mathrm{rad}^{2} \mathrm{~s}^{-2} \mathrm{~W}^{-1} g^{-1}$, and $0.62 \mathrm{~ms}$ for $c_{1}, c_{2}$, and $t_{0}$, respectively. By repeating this fitting procedure for different microwave frequencies $f$, we verify that $t_{0}$ remains largely unchanged while $c_{1}$ and $c_{2}$ attain peaks at the microwave resonance, provided that the gas pressure is kept constant. In Fig. 4, we plot the dependence of $F_{\mu \text { wave }}=c_{1} I_{0}$ on microwave frequency ranging from 13.8 to $14.8 \mathrm{GHz}$ at $2.5 \times 10^{-4}$ torr as purple circles. For measurements beyond this range of microwave frequency, the fitting did not return consistent results because of low signal levels. At this gas pressure, $F_{\text {thermal }}$ and $F_{\mu \text { wave }}$ are comparable in magnitude, with $F_{\text {thermal }} \sim 1.1 F_{\mu \text { wave }}$, throughout the range of microwave frequencies. The good agreement with the numerical calculations and with the measurements at $8.6 \times 10^{-3}$ torr (where thermal effects are negligible) supports the validity of the model.

In summary, we have experimentally demonstrated that a strong repulsive radiation force between the two plates can be induced by the incident microwave radiation. By carefully distinguishing the direct EM induced force and the indirect photothermal force, the EM force is found to be two orders of magnitude larger than the ordinary radiation force. Our work opens the possibility for exploiting EM induced forces in micromechanics, such as the construction of tunable and nonlinear metamaterials, in the microwave regime. With qualitatively similar enhancement of the photon pressure already predicted theoretically for plasmonic cavities at optical frequencies [18], it is promising that further progress can be made in different frequency regimes by exploiting resonance-enhanced EM forces in metamaterials made up of an array of resonating units.
Z. M. and Z. H. H. contributed equally to this work. This work is supported by Grants No. HKUST2/CRF/11G and No. AoE/P-02/12 from the Research Grants Council of Hong Kong SAR. Z.H.H. is also supported by the NSFC (Grant No. 11304215) and the NSF of Jiangsu Province (Grant No. BK20130281).

*hochan@ust.hk

[1] J. B. Pendry, Contemp. Phys. 45, 191 (2004).

[2] R. A. Shelby, D. R. Smith, and S. Schultz, Science 292, 77 (2001).

[3] J. B. Pendry, Phys. Rev. Lett. 85, 3966 (2000).

[4] D. O. S. Melville, R. J. Blaikie, and C. R. Wolf, Appl. Phys. Lett. 84, 4403 (2004).

[5] X. Zhang and Z. W. Liu, Nat. Mater. 7, 435 (2008).

[6] H. Y. Chen, C. T. Chan, and P. Sheng, Nat. Mater. 9, 387 (2010).

[7] M. Lapine, I. V. Shadrivov, D. A. Powell, and Y. S. Kivshar, Nat. Mater. 11, 30 (2011).

[8] A. D. Boardman, V. V. Grimalsky, Y.S. Kivshar, S. V. Koshevaya, M. Lapine, N. M. Litchinitser, V. N. Malnev, M. Noginov, Y. G. Rapoport, and V. M. Shalaev, Laser Photonics Rev. 5, 287 (2011).

[9] P. Y. Chen, M. Farhat, and A. Alu, Phys. Rev. Lett. 106, 105503 (2011).

[10] J. Y. Ou, E. Plum, L. Jiang, and N. I. Zheludev, Nano Lett. 11, 2142 (2011).

[11] H. Tao, A. C. Strikwerda, K. Fan, W. J. Padilla, X. Zhang, and R. D. Averitt, Phys. Rev. Lett. 103, 147401 (2009).

[12] M. Lapine, M. Gorkunov, and K. H. Ringhofer, Phys. Rev. E 67, 065601 (2003).

[13] A. A. Zharov, I. V. Shadrivov, and Y. S. Kivshar, Phys. Rev. Lett. 91, 037401 (2003).

[14] G. Volpe, R. Quidant, G. Badenes, and D. Petrov, Phys. Rev. Lett. 96, 238101 (2006).

[15] M. Ploschner, M. Mazilu, T. F. Krauss, and K. Dholakia, J. Nanophoton. 4, 041570 (2010).

[16] R. Zhao, P. Tassin, T. Koschny, and C. M. Soukoulis, Opt. Express 18, 25665 (2010).

[17] S. B. Wang, J. Ng, H. Liu, H. H. Zheng, Z. H. Hang, and C. T. Chan, Phys. Rev. B 84, 075114 (2011).

[18] H. Liu, J. Ng, S. B. Wang, Z. F. Lin, Z. H. Hang, C. T. Chan, and S. N. Zhu, Phys. Rev. Lett. 106, 087401 (2011).

[19] J. Kohoutek, D. Dey, A. Bonakdar, R. Gelfand, A. Sklar, O. G. Memis, and H. Mohseni, Nano Lett. 11, 3378 (2011).

[20] Y. R. He, S. L. He, J. Gao, and X. D. Yang, Opt. Express 20, 22372 (2012).

[21] J. Zhang, K. F. MacDonald, and N. I. Zheludev, Phys. Rev. B 85, 205123 (2012).

[22] C. H. Metzger and K. Karrai, Nature (London) 432, 1002 (2004).

[23] J. J. Xiao and C. T. Chan, J. Opt. Soc. Am. B 25, 1553 (2008).

[24] S. B. Wang, H. H. Zheng, J. J. Xiao, Z. F. Lin, and C. T. Chan, Int. J. Comp. Mat. Sci. Eng. 1, 1250038 (2012).

[25] COMSOL Multiphysics 3.5 (www.comsol.com).

[26] See, for instance, J. D. Jackson, in Classical Electrodynamics (Wiley, New York, 1998), 3rd ed., p. 261. 